This is an electronic reprint of the original article. This reprint may differ from the original in pagination and typographic detail.

Author(s): Merikoski, Juha

Title: $\quad$ Accelerated transport and growth with symmetrized dynamics

Year: $\quad 2013$

Version:

Please cite the original version:

Merikoski, J. (2013). Accelerated transport and growth with symmetrized dynamics.

Physical Review E, 88(6), Article 062137.

https://doi.org/10.1103/PhysRevE.88.062137

All material supplied via JYX is protected by copyright and other intellectual property rights, and duplication or sale of all or part of any of the repository collections is not permitted, except that material may be duplicated by you for your research use or educational purposes in electronic or print form. You must obtain permission for any other use. Electronic or print copies may not be offered, whether for sale or otherwise to anyone who is not an authorised user. 


\title{
Accelerated transport and growth with symmetrized dynamics
}

\author{
Juha Merikoski* \\ Department of Physics, University of Jyväskylä, P. O. Box 35, FI-40014 Jyväskylä, Finland \\ (Received 25 June 2013; revised manuscript received 14 October 2013; published 23 December 2013)
}

\begin{abstract}
In this paper we consider a model of accelerated dynamics with the rules modified from those of the recently proposed [Dong et al., Phys. Rev. Lett. 109, 130602 (2012)] accelerated exclusion process (AEP) such that particle-vacancy symmetry is restored to facilitate a mapping to a solid-on-solid growth model in $1+1$ dimensions. In addition to kicking a particle ahead of the moving particle, as in the AEP, in our model another particle from behind is drawn, provided it is within the "distance of interaction" denoted by $\ell_{\max }$. We call our model the doubly accelerated exclusion process (DAEP). We observe accelerated transport and interface growth and widening of the cluster size distribution for cluster sizes above $\ell_{\max }$, when compared with the ordinary totally asymmetric exclusion process (TASEP). We also characterize the difference between the TASEP, AEP, and DAEP by computing a "staggered" order parameter, which reveals the local order in the steady state. This order in part explains the behavior of the particle current as a function of density. The differences of the steady states are also reflected by the behavior of the temporal and spatial correlation functions in the interface picture.
\end{abstract}

DOI: 10.1103/PhysRevE.88.062137

PACS number(s): 05.40.-a, 02.50.Ey, 81.15.Aa

\section{INTRODUCTION}

The totally asymmetric exclusion process (TASEP) and the related zero-range process have become paradigmatic models of low-dimensional nonequilibrium dynamics [1-5]. Macroscopic quantities of interest, including the current through the system and its fluctuations, as well as configurational properties, such as the dynamics of the largest cluster and the condensation transition, have been of a renewed interest for various generalizations [6-17] of the basic TASEP model, many of them interesting, e.g., from the point of view of RNA transcription, jamming of traffic flow, phase separation, and growth phenomena.

Very recently, an accelerated exclusion process (AEP) was developed and analyzed by Dong et al. [16,17]. In the AEP a particle hopping into a (particle) cluster "kicks" the frontmost particle of that cluster one site forward. The kicked particle cannot cause any further kicks. In Refs. [16] and [17] this is argued to lead via condensation to the existence of a novel "unit velocity" (UV) phase with the particle current $J=1-\rho$ for particle densities $\rho>1 / 2$. The value of $\rho$ for the emergence of the UV phase depends on the value of the control parameter $\ell_{\max }$, the range (see below for its definition) of the kick interaction. In the definition of the AEP model the particle-vacancy symmetry is broken, but the dynamics can be nevertheless successfully understood by considering the concerted motion of vacancies for large values of $\ell_{\max }$ [17]. In the present work we further generalize the TASEP to include a "draw" (from behind) interaction, to restore the particle-vacancy symmetry. In addition to providing one generalization of the particle model, a major motivation for this is to enable mapping to a solid-on-solid interface growth model. The related interface models have been extensively studied for their scaling properties [4,18-21] We call our model the doubly accelerated exclusion process (DAEP).

\section{MODEL AND NUMERICAL METHODS}

The dynamical rules of the DAEP model and its mapping onto a solid-on-solid (SOS) model are shown in Fig. 1. We consider a one-dimensional lattice of $L$ sites $i$ with occupation number $n_{i}=0,1$ and periodic boundary conditions $n_{i+L}=n_{i}$. The total number of particles, $N=\sum_{i} n_{i}$, is kept constant, with $\rho=N / L$ giving the particle density.

The hopping rules of the DAEP are as follows: A particle at site $i=1,2, \ldots, L$ hops into a neighboring empty site on the right with unit probability in time. Into the ordinary TASEP model two possible adjoint moves controlled by the parameter $\ell_{\max }$ are added: If the particle hops into an existing particle cluster with length $\ell_{p} \leqslant \ell_{\max }$ it will kick the frontmost particle of that cluster one site ahead (this is the AEP rule). In addition, if the hopping particle has immediately behind it a vacancy cluster of length $\ell_{v} \leqslant \ell_{\max }$, it will draw the frontmost particle of the preceding particle cluster one site ahead; see Fig. 1(a). The particles kicked or drawn will themselves cause no further particle moves.

The DAEP model is mapped onto a SOS model as indicated in Fig. 1(b). An occupied site, $n_{i}=1$, in the DAEP corresponds to a step down in the SOS model and a vacant site, $n_{i}=0$, corresponds to a step up. The interface configuration is given by a height function $h_{j}$, where $j=1,2, \ldots, L$ is the index of a column. The configurations in Figs. 1(a) and 1(b) correspond to each other one to one, except for the overall height of the interface configuration, which can be shifted by a constant value. Since all the particle moves (hop, kick, draw) are directed to the right, each of them corresponds to a local upward move of the interface by two height units, as indicated in Fig. 1(b) by the $1 \times 2$ blocks landing on the interface. In this way the BSCOS rule $h_{j+1}-h_{j}= \pm 1$ is preserved [18]. The difference in height between adjacent columns can also be interpreted as a classical spin- $1 / 2$ variable. In the mapping, the locations in the horizontal direction of interface columns are between two adjacent sites of the particle model.

We note that since $\ell_{\max }$ is the same for both kick and draw processes, the left-right symmetry is preserved in the interface model. A kick means adsorption onto a site right of

*juha.t.merikoski@jyu.fi 
(a)

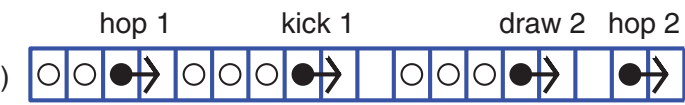

(b)

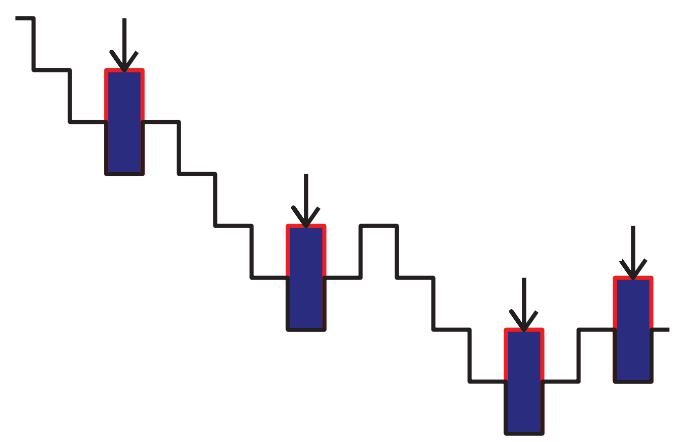

FIG. 1. (Color online) (a) The moves in the DAEP model and (b) the corresponding growth events in the body-centered solid-onsolid (BCSOS) model. In (a) moving particles are indicated by solid circles, and other particles by open circles (otherwise there is no difference between them). The hop indicated by "hop 1" causes the move indicated by "kick 1 " if $\ell_{\max } \geqslant 4$ and the (independent) hop indicated by "hop 2" causes the move "draw 2 " if $\ell_{\max } \geqslant 2$.

the landing site corresponding to the hop, and a draw means in the interface picture adsorption onto a site left of the landing site corresponding to the hop. In the particle model this means particle-vacancy symmetry such that a particle system with density $\rho$ corresponds to a vacancy system with density $1-\rho$.

Yet another mapping of the AEP model exists, namely, that onto a mass-transport process (MTP) [16,17], which is a generalization of the zero-range process (ZRP). Within the MTP picture hops and kicks are easily handled, with a hop + kick effectively leading to one hop of length 2 in the MTP, i.e., to an effective interaction of short range in the MTP coordinates. Adding the draw process leads to interaction over the preceding vacancy cluster in the DAEP, which corresponds to effective interaction over up to $\ell_{\text {max }}$ adjacent empty sites in the MTP picture. Thus the MTP description for the DAEP would become complicated and will not be used here.

\section{RESULTS}

In Fig. 2 we show simulation results for the particle current $J$ in the DAEP model for several values of $\ell_{\max }$. In contrast with the AEP [16,17], by construction, the behavior in the DAEP is symmetric such that $J(\rho)=J(1-\rho)$. This is due to the particle-vacancy symmetry of DAEP.

For the calculations of the current, we have used both the step initial conditions with all particles initially confined in a single cluster and the totally disordered initial state, with similar results for the steady state. We do not observe the unit-velocity-type phase for any initial conditions. A typical length of the simulations was of the order of 200000 Monte Carlo steps (trial hops) per particle with random update of sites and we sampled the steady state reached after 100000 steps. As in Refs. [16,17], the system size is $L=1000$ in all simulation results shown.

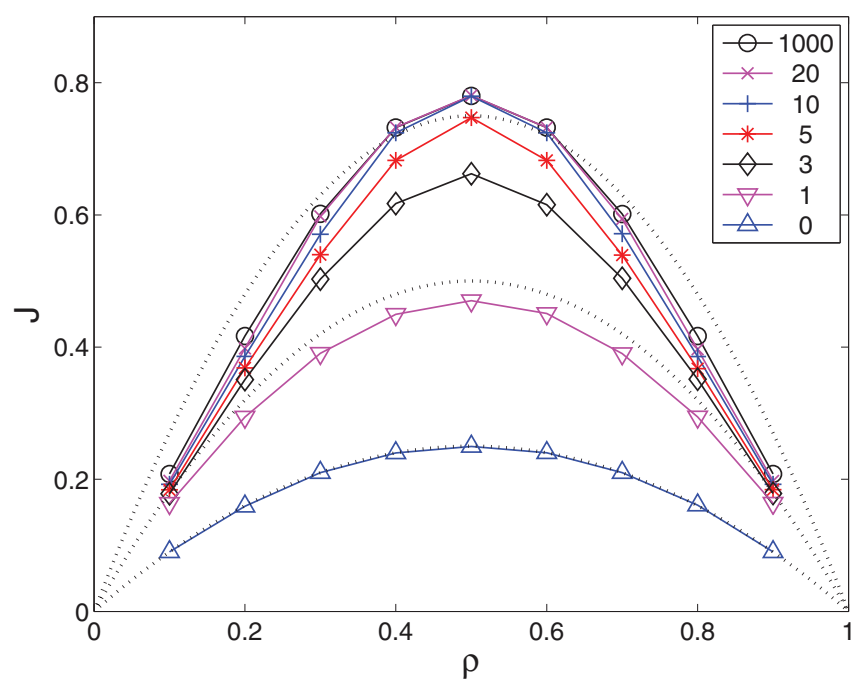

FIG. 2. (Color online) The average current $J$ as a function of particle density $\rho$ for $\ell_{\max }=0,1,3,5,10,20,1000$ in the DAEP model with $L=1000$. The three dotted curves denote $J_{\text {tasep }}, 2 J_{\text {tasep }}$, and $3 J_{\text {tasep }}$, from bottom to top.

As in the AEP, also in the DAEP transport is accelerated such that $J$ is larger than $J_{\text {tasep. }}$. For $\ell_{\max }>0$ at and close to $\rho=1 / 2$ with large $\ell_{\max }$ the current is even larger than the first mean-field (MF) guess: We find $J>3 J_{\text {tasep }}$, where $J_{\text {tasep }}=\rho(1-\rho)$ is the current in the ordinary TASEP in the thermodynamic limit [2]. In addition to this comparison with the TASEP, we have attempted several functional forms for $J(\rho)$ preserving the particle-vacancy symmetry, but such forms cannot be justified by approximations derived from MTP such as the one in Refs. [16,17], since the MTP description is particle-vacancy asymmetric. Thus $J \approx 3 J_{\text {tasep }}$ for large $\ell_{\max }$ remains our best approximation. In particular, MTPMF-type approximations [cf. Eq. (11) of Ref. [17]] do not work because of, e.g., the asymmetric factor $(1-\rho)$ in the the MTP-ASEP back transformation.

The shape of the curves $J=J(\rho)$ is nevertheless different from the shape of $J_{\text {tasep }}$. For large $\ell_{\max }$ and small $\rho$ they are almost linear up to $\rho<1 / 4$ and, correspondingly, for $\rho>3 / 4$. A reasonably good approximation for large $\ell_{\max }$ is given by $J(\rho<1 / 4) \approx 2 \rho$ and $J(\rho>3 / 4) \approx 2(1-\rho)$. Interestingly, the latter is twice the current in the unit-velocity phase in the AEP. No turning point is observed in the $J=J(\rho)$ curves; cf. Fig. 3 in [17].

The slope $\partial J / \partial \rho \approx 2$ for small densities is understood most easily by considering the cases $N=2$ and $N=3$. For $N=2$ the state where the distance between the two particles remains 2 is an absorbing state, i.e., a local particle configuration 0010100 ( 0 refers to an empty site and 1 to an occupied site) has two possibilities for a configuration change, when both kick and draw processes are effective: Either the frontmost particle is chosen and hops, drawing the trailing one with it, or the trailing particle hops, kicking the frontmost particle one step ahead. Both of these processes lead to the configuration 0001010 and a contribution of two jumps to the current; thus $J=2 \rho$. For high densities a similar argument 
for two vacancies (holes) leads to the average jump rate 2 and $J=2(1-\rho)$.

For $N=3$ one can start the discussion by considering an initial state 001010100, where there are three possibilities: If the frontmost particle hops, it draws the second one with it, leading to 001001010. If the particle in the center hops, all three particles move, leading to 000101010. If the last one hops it kicks the particle in the middle, leading to 000101100. From the last one of these there are only two possibilities: either the frontmost particle hops, leading to one jump only and the configuration 0000101010, or the last one hops, leading to two jumps and the same configuration 0000101010. On the average, these processes lead to approximately two jumps for each hop. In the case $N=3$ one should note that it is not possible for the first particle alone to escape the second one; it will draw the second particle with it (with a vacant site between them). After that the third particle still has an effective jump rate of 2 , either by hopping or being drawn by the second particle, if it is within the range $\ell_{\max }$. Thus the system of the three particles is loosely bound, with the average particle jump rate close to 2 .

The discussion of the cases $N=2$ and $N=3$ also explains why $\partial J / \partial \rho \approx 2$ remains valid for larger densities for larger values of $\ell_{\max }$, i.e., for effective interactions of longer range. The effective attraction tends to build and stabilize local configurations of the type considered above.

As a partial explanation of the difference between the AEP and DAEP for larger $N$, we note also that in the DAEP the "balance" of, e.g., the largest particle cluster becomes different from that in the AEP. In the DAEP there are two ways particles can come into the cluster and two ways (not just the one in the AEP) out of it.

The particle and vacancy cluster size distributions are interesting in that there first is an exponential decay with one value of the decay parameter for cluster size $s<\ell_{\max }$, after which there is a slower exponential decay for $s>\ell_{\max }$; see Fig. 3(a). A finite value of $\ell_{\max }$ thus induces a length scale in the system and widens the cluster size distribution above it. The vacancy cluster distributions (not shown) for density $\rho$ are similar to particle cluster distributions for density $1-\rho$. Figure 3(b) reveals one striking feature: The distributions are nonmonotonic with a dip (the same is observed for vacancy clusters at density $1-\rho)$. The increase of $f_{s}(p)$ just above $s=\ell_{\max }$ can be understood as follows: the kick process no longer contributes to the exit rate from the cluster, thereby changing its balance equation.

Let us next turn to the interface picture with $\rho=1 / 2$. We have monitored the surface roughness in the transient state and spatial and temporal correlations in the steady state. For the transient dynamics, we used initial conditions corresponding to a "flat" interface or $\rho=1 / 2$ with even-numbered sites filled. This choice of $\rho$ gives a globally untilted interface. Note that there is an intrinsic roughness even in the flattest possible configuration, consisting of alternating up and down steps because of the condition $h_{j+1}-h_{j}= \pm 1$.

The interface width or roughness is measured by [18]

$$
W(t)=\left\langle\left[h_{j}(t)-\bar{h}(t)\right]^{2}\right\rangle^{1 / 2} .
$$

Here $\langle\cdot\rangle$ denotes an ensemble average, $\bar{h}(t)$ is the configuration average, and $t$ is the real time measured from the start of the
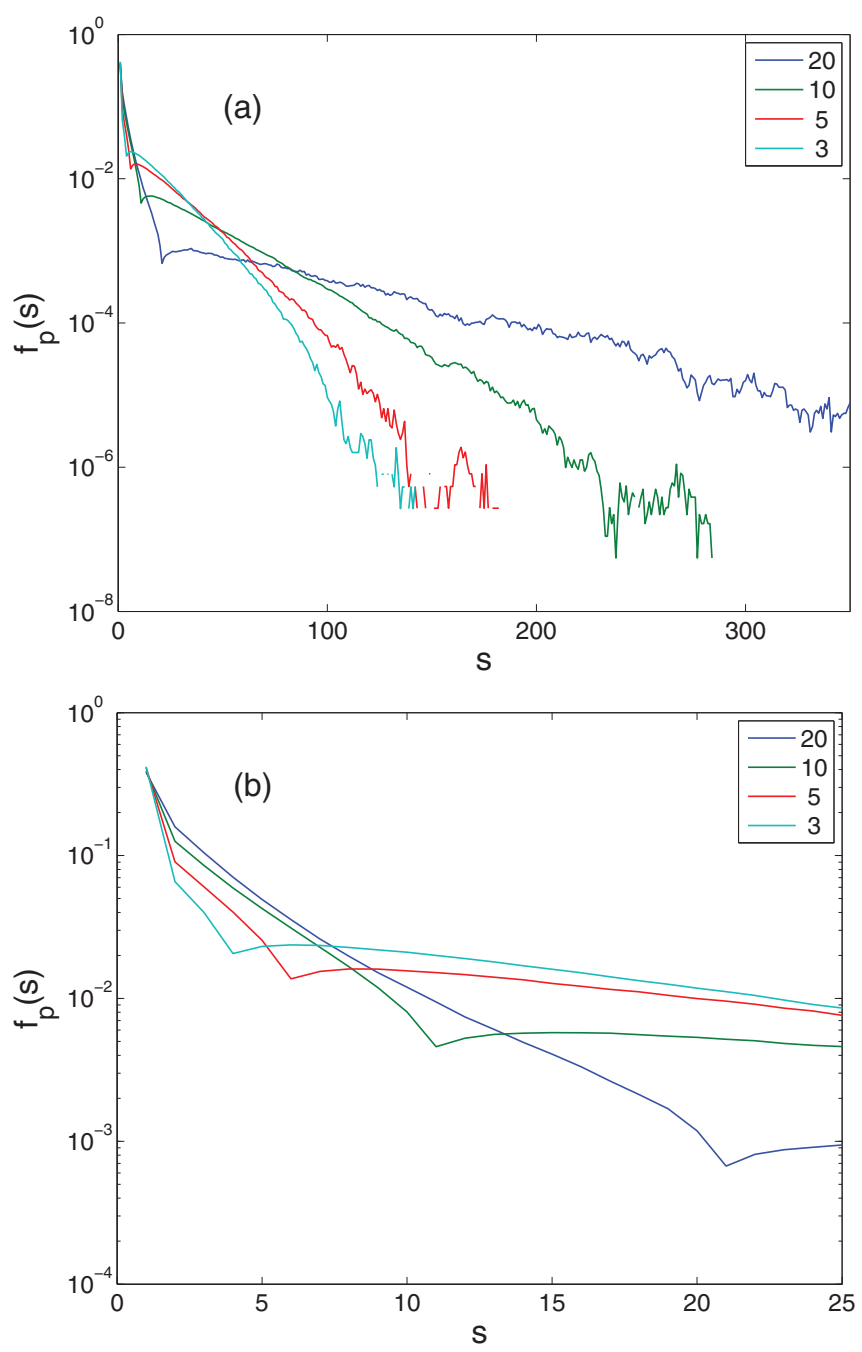

FIG. 3. (Color online) (a) The particle cluster size distributions $f_{p}(s)$ for $\rho=0.9$ and $\ell_{\max }=3,5,10,20$. (b) The same data for small cluster sizes $s$. In both figures a cusp and change of the decay parameter of the apparent exponential decay are observed just above $s=\ell_{\max }$.

simulation. The intrinsic roughness is handled by calculating $W(t)$ separately for even and odd sites. As the spatial heightheight correlation function, which we measure in the steady state, we take

$$
C(r)=\left\langle\left[h_{j+r}(t)-h_{j}(t)\right]^{2}\right\rangle^{1 / 2},
$$

where $r$ is taken to be either even or odd to avoid oscillations due to the intrinsic roughness. As the temporal one we take

$$
C(t)=\left\langle\left[h_{j}\left(t^{\prime}+t\right)-h_{j}\left(t^{\prime}\right)\right]^{2}\right\rangle^{1 / 2},
$$

where $t$ is the time difference (in the steady state).

The transient behavior of the interface width for the TASEP, AEP, and DAEP at $\rho=1 / 2$ are shown in Fig. 4(a). For long times, corresponding to the steady state, the roughness $W^{\text {ss }}$ differs slightly for the three models such that as computed from the simulation data (not easily discernible from the plot) one finds $W_{\text {tasep }}^{\text {ss }}<W_{\text {daep }}^{\text {ss }}<W_{\text {aep }}^{\text {ss }}$. This is understandable as follows: The steady state for the TASEP is fully disordered 

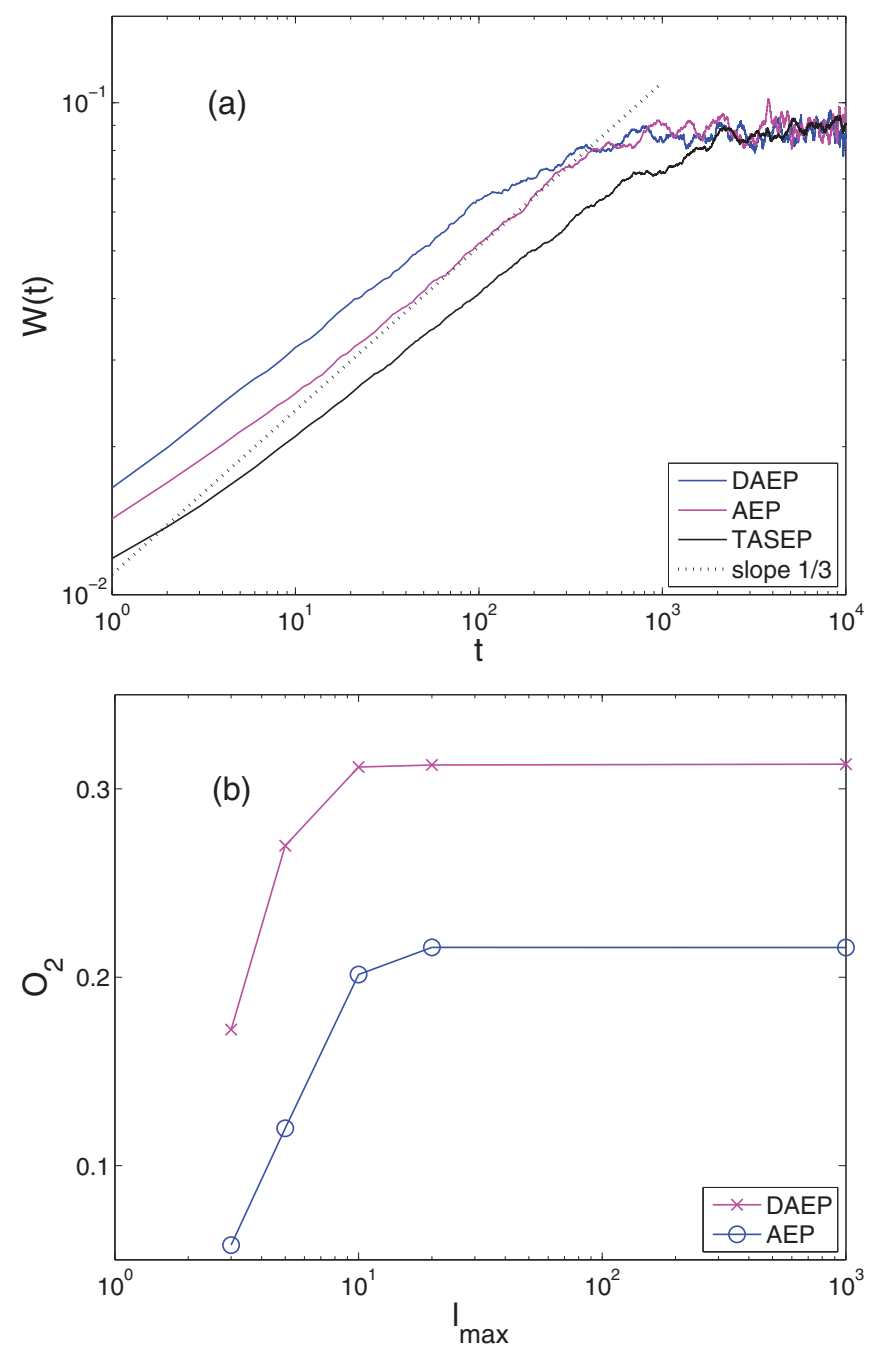

FIG. 4. (Color online) (a) Transient behavior of the interface width $W(t)$ for $\rho=1 / 2$ starting from an initially flat interface. Solid curves from top to bottom correspond to the DAEP, AEP, and TASEP, respectively, for the DAEP and AEP with $\ell_{\max }=10$. The dotted line corresponds to $W(t) \sim t^{1 / 3}$. (b) The stationary value of the order parameter $O_{2}$ for $\rho=1 / 2$ as a function of $\ell_{\max }$ for the DAEP and AEP. For $\ell_{\max }=0$ both models reduce to the TASEP with $O_{2}=0$.

[2]; AEP dynamics induces additional structure in the configurations [17] and so does (somewhat less) the DAEP.

A major second difference when comparing the DAEP with the standard TASEP turns out to be the short-range order, as measured by the "staggered" order parameter $\mathrm{O}_{2}$ defined most conveniently in the interface picture as

$$
O_{2}=\left\langle\left(h_{j}-h_{j+1}\right)\left(h_{j+2}-h_{j+1}\right)\right\rangle .
$$

In the particle picture this is 1 for local two-site configurations 01 and 10 and -1 for 00 and 11 . For the fully disordered stationary state of the TASEP one has $\mathrm{O}_{2}=0$. For the DAEP the local configurations 01 and 10 are favored by the dynamics (cf. the discussion of two-particle and three-particle cases above) such that $O_{2}>0$ and increases with increasing $\ell_{\max }$. The role of $\ell_{\max }$ is to increase the effective attractive interaction such that it tends to bring particles together in 0101010-like configurations. For the AEP also $\mathrm{O}_{2}>0$, but it increases
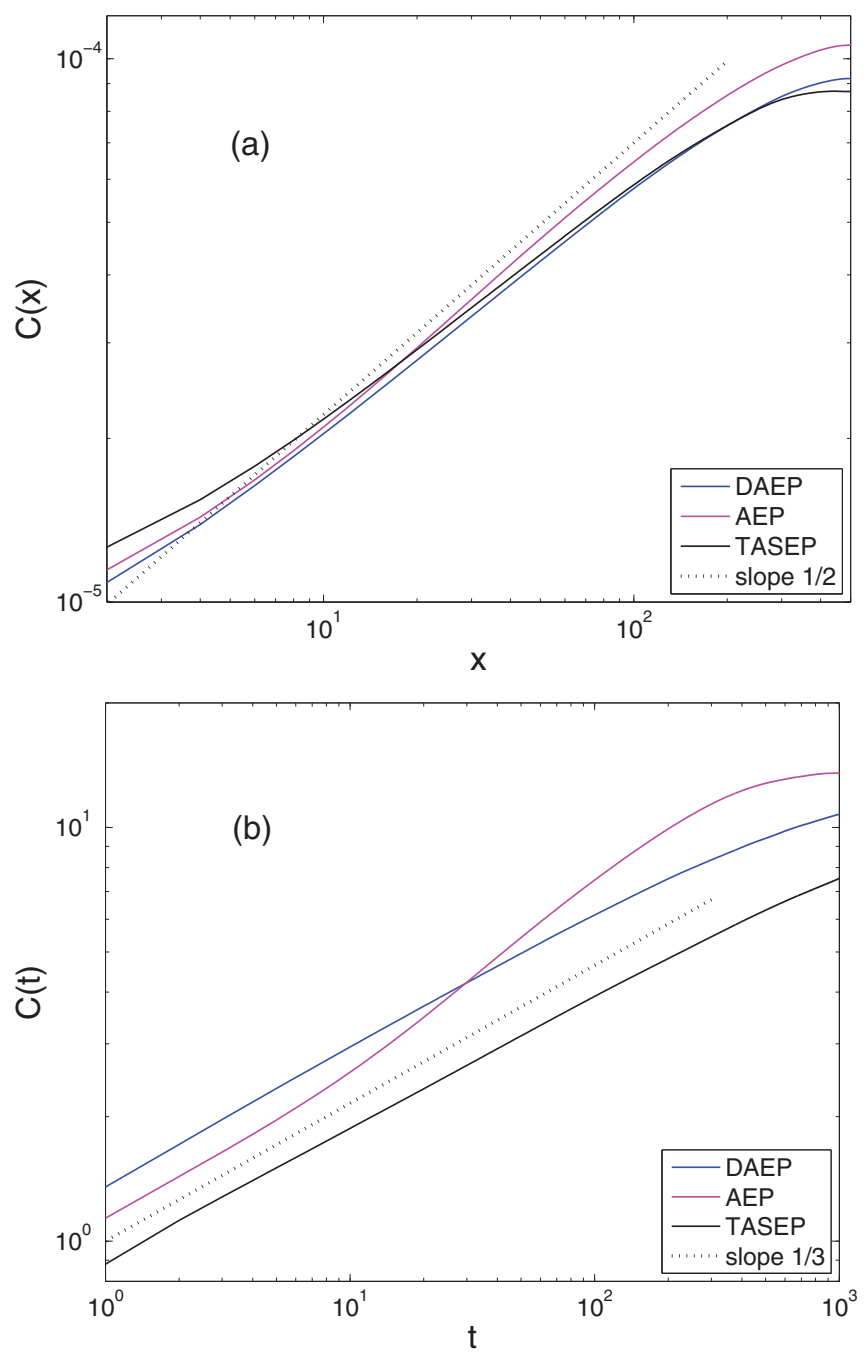

FIG. 5. (Color online) (a) The spatial and (b) the temporal correlation functions in the steady state. In (a) for large distances and in (b) for large times the largest values are obtained for the AEP and the smallest ones for the TASEP. For all cases $\rho=1 / 2$ and for the DAEP and AEP $\ell_{\max }=10$.

much more slowly at small values of $\ell_{\max }$. This local order causes the rapid initial decay of the cluster (vacancy) size distribution for small clusters (vacancies) up to $s=\ell_{\max }$, i.e., up to distances where the kick and draw processes are effective. It leads to a (partly) faceted interface and enhances the particle current as discussed above. Data for $\mathrm{O}_{2}$ are shown in Fig. 4(b).

From Fig. 5 we find that the dynamics of the interface in the steady state is accelerated such that the temporal correlation function initially rises most rapidly in the DAEP but is overtaken by the AEP for large times. The scale defined by $\ell_{\max }$ and visible in the cluster distributions is, however, not directly discernible in the correlation functions. This can be understood by noting the fast initial decay of the cluster distributions: The particle clusters with $s>\ell_{\max }$ have a relatively small probability and their distribution is wide. At large distances and long times the correlation functions are again ordered such that the value for the AEP is larger than that for the DAEP, the value for the TASEP being the smallest. In Figs. 4 and 5 the 

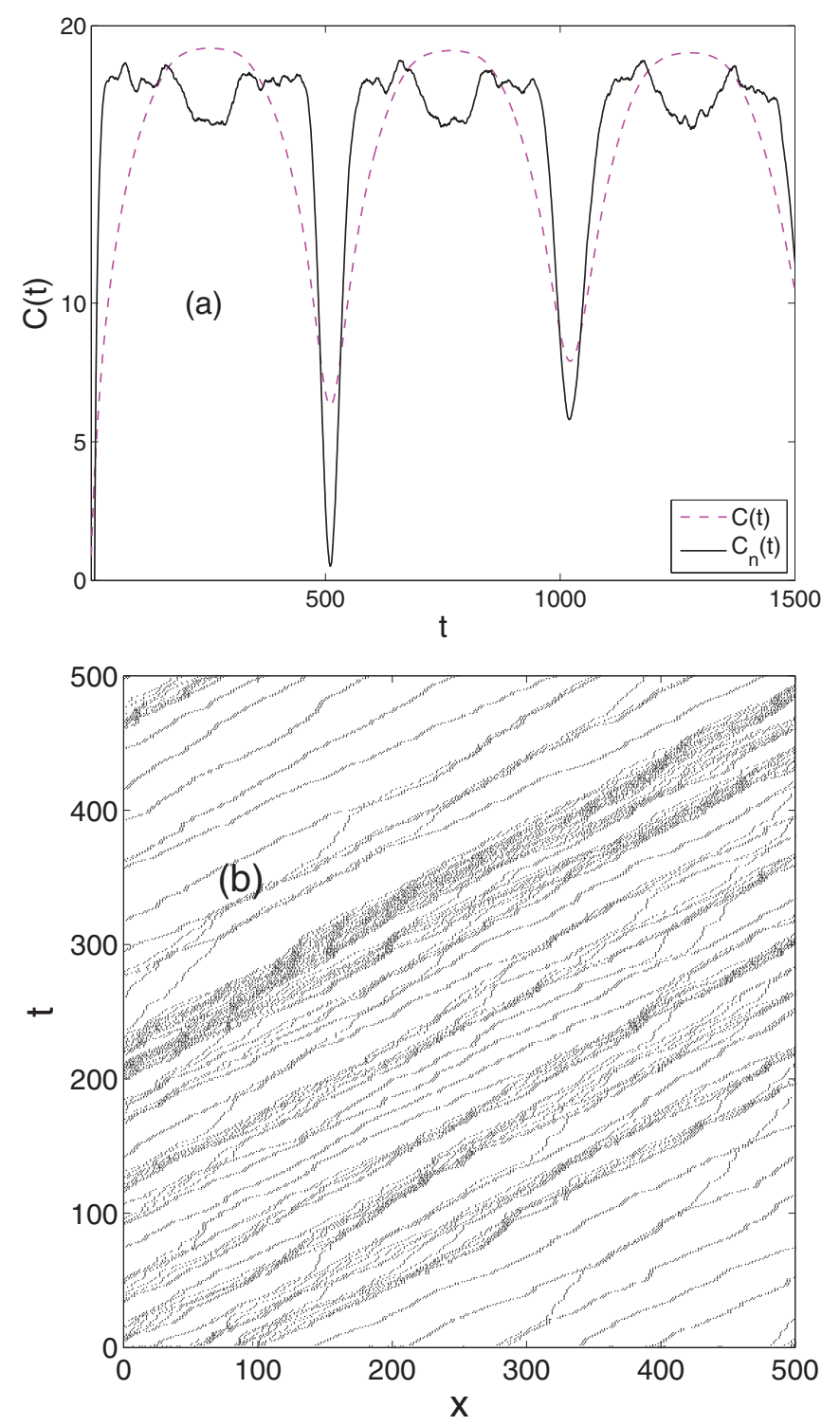

FIG. 6. (Color online) (a) The temporal height-height correlation function $C(t)$ for $\rho=1 / 10$ with $\ell_{\max }=10$ and the corresponding (scaled and shifted) particle-particle correlation function $C_{n}(t)$ in the steady state. (b) Snapshots of a part of a particle configuration in the DAEP. Here $L=1000$, but for clarity only part of the configuration is shown.

dotted lines correspond to the expected Kardar-Parisi-Zhang scaling behavior [18] for intermediate times and distances.

It is also possible to study correlations for values of the density other than $\rho=1 / 2$. In the interface picture this corresponds to a globally tilted interface. There we observe oscillatory behavior in $C(t)$ as shown in Fig. 6(a) for $\rho=0.1$. For comparison we plot the particle-particle (actually the spin-spin) correlation function $C_{n}(t)$, in the steady state:

$$
\begin{aligned}
C_{n}(t)= & \left\langle\left[2 n_{i}\left(t+t^{\prime}\right)-1\right]\left[2 n_{i}\left(t^{\prime}\right)-1\right]\right\rangle \\
& -\left\langle 2 n_{i}\left(t+t^{\prime}\right)-1\right\rangle\left\langle 2 n_{i}\left(t^{\prime}\right)-1\right\rangle,
\end{aligned}
$$

which displays similar oscillations, yet much weaker (there is a large vertical scaling factor in the plot). From the snapshots in Fig. 6(b) we can deduce a "velocity" of $v \approx 2$ (in natural units) and a related time scale $\tau=L / v \approx 500$. This is roughly the period of oscillation in $C(t)$ and $C_{n}(t)$. For comparison, the period of oscillation (figure not shown) for $\rho=0.4$ is roughly 900, with the apparent velocity still around $v \approx 2$. These results for the velocity can be compared with the prediction for the particle velocity from the values obtained from the current: taking $v_{p}=J / \rho$ we have $v_{p}(\rho=0.4) \approx 1.8$ and $v_{p}(\rho=0.1) \approx 1.9$. The oscillations in $C(t)$ get weaker the closer to one-half the density is. We conclude that local disturbances in density live for a long time and can propagate over the whole particle system, thus producing oscillations in $C(t)$ in the corresponding long time scales.

In Fig. 6(b) we also see typical space-time trajectories corresponding to the local ordering: For example, at $x \approx 320$ and $t \approx 20$ we see the last particle of a group of three to be left behind. The remaining two particles continue together with average velocity 2 . The particle left behind propagates with a slower velocity and eventually it is adsorbed by another pair of particles at $x \approx 350$ and $t \approx 50$. Several similar sequences are seen in the figure. Within the denser patch close to the middle of the figure the density of vacancies is also close to one-half. Thus the average velocity is close to 2 and the current $J \approx 2 \rho$.

\section{CONCLUSIONS}

To conclude, we have studied a generalization of the TASEP model, which we called the DAEP model. This is a symmetrized version of the AEP model [16,17] and corresponds to a solid-on-solid interface model with dynamics including long-range interactions up to a scale given by the parameter $\ell_{\max }$. Including an additional kind of move, a draw, in the AEP model containing a hop and a kick, is found to change the unit-velocity phase observed in the AEP for high density. For low and high densities in the DAEP we find $J \approx$ $2 \rho$ and $J \approx 2(1-\rho)$, respectively. The draw process provides a way for the "fluid phase" to rip off a particle from a large cluster or "condensate," thus modifying the balance equation for large clusters: There are now two ways in and two ways out (cf. the two ways in and one way out in the AEP) and no sign of a condensate is seen. More importantly, the behavior of the current at low and high density was explained by considering prominent few-particle configurations, corresponding to local order measured by an order parameter. A peculiarity of the DAEP is that the dynamics strongly favors configurations with adjacent vacant and occupied sites. The different jump mechanisms are most effective at half filling, resulting in a pronounced maximum of the total current there, the current exceeding three times that of the TASEP. We then studied the correlations in the corresponding solid-on solid model, and observed accelerated dynamics. No simple, working analytic approximation appears to be available for the cluster size distributions of the DAEP, because the effective interaction due to the draw process is of long range in an MTP, in which the the kick process becomes short range, and vice versa. For the same reason the model eludes solution by Bethe ansatz. In possible further studies, the conditions for the existence of real condensation transition and the behavior of the condensate in various modified TASEP models, including those with open boundaries, are of interest $[9,14]$. 
[1] F. Spitzer, Adv. Math. 5, 246 (1970).

[2] H. Spohn, Large Scale Dynamics of Interacting Particles (Springer, New York, 1991).

[3] B. Schmittmann and R. K. P. Zia, in Phase Transitions and Critical Phenomena, edited by C. Domb and J. Lebowitz, Vol. 17 (Academic Press, London, 2001).

[4] M. R. Evans and T. Hanney, J. Phys. A 38, R195 (2005).

[5] T. Chou, K. Mallick, and R. K. P. Zia, Rep. Prog. Phys. 74, 116601 (2011).

[6] T. Antal and G. M. Schütz, Phys. Rev. E 62, 83 (2000).

[7] G. M. Schütz, in Phase Transitions and Critical Phenomena, edited by C. Domb and J. Lebowitz, Vol. 19 (Academic Press, London, 2001).

[8] M. R. Evans, T. Hanney, and S. N. Majumdar, Phys. Rev. Lett. 97, 010602 (2006).

[9] O. Hirschberg, D. Mukamel, and G. M. Schütz, Phys. Rev. Lett. 103, 090602 (2009).

[10] A. Gabel, P. L. Krapivsky, and S. Redner, Phys. Rev. Lett. 105, 210603 (2010)
[11] A. Gabel and S. Redner, J. Stat. Mech.: Theory Exp. (2011) P06008.

[12] B. Waclaw and M. R. Evans, Phys. Rev. Lett. 108, 070601 (2012).

[13] S. Grosskinsky, F. Redig, and K. Vafayi, Electron. J. Probab. 18, 1 (2013).

[14] O. Hirschberg, D. Mukamel, and G. M. Schütz, Phys. Rev. E 87, 052116 (2013)

[15] P. L. Krapivsky, J. Stat. Mech.: Theory Exp. (2013) P06012.

[16] J. Dong, S. Klumpp, and R. K. P. Zia, Phys. Rev. Lett. 109, 130602 (2012).

[17] J. Dong, S. Klumpp, and R. K. P. Zia, Phys. Rev. E 87, 022146 (2013).

[18] A.-L. Barabasi and H. E. Stanley, Fractal Concepts in Surface Growth (Cambridge University Press, Cambridge, 1995).

[19] J. Krug, Adv. Phys. 46, 139 (1997).

[20] M. Pradas, J. M. López, and A. Hernández-Machado, Phys. Rev. E 76, 010102(R) (2007).

[21] F. D. A. Aarao Reis, Phys. Rev. E 84, 031604 (2011). 